DOI: https://doi.org/10.11144/Javeriana.umed61-4.fibr

\title{
Fibrosis pulmonar en infección por SARS- CoV-2: ¿qué sabemos hasta ahora? ¿Qué podemos esperar?
}

\section{Pulmonary Fibrosis in SARS-CoV2 Infection. What we Know? What we could Expect?}

July VIANNETh ToRRES-GonzÁLEZ

Médica internista neumóloga, Hospital Universitario San Ignacio, Bogotá, Colombia

Juan David Botero

Fellow en Neumología, Pontificia Universidad Javeriana-Hospital Universitario San
Ignacio, Bogotá, Colombia

Carlos Andrés Celis-Preciado

Departamento de Medicina Interna, Facultad de Medicina, Pontificia Universidad Javeriana, Bogotá, Colombia. Médico internista neumólogo, Hospital Universitario

San Ignacio, Bogotá, Colombia María José Fernández

Departamento de Medicina Interna, Facultad de Medicina, Pontificia Universidad Javeriana, Bogotá, Departamento de Ciencias Fisiológicas, Facultad de Medicina, Pontificia Universidad Javeriana, Médica internista neumóloga, Hospital Universitario San Ignacio, Bogotá, Colombia Claudio Villaguirán Departamento de Medicina Interna, Facultad de Medicina, Pontificia Universidad Javeriana, Bogotá, Médico internista neumólogo, Hospital Universitario San Ignacio, Bogotá, Colombia Olga Milena García

Médica internista neumóloga, Hospital Universitario San Ignacio, Bogotá, Colombia Iván Solarte

Departamento de Medicina Interna, Facultad de Medicina, Pontificia Universidad Javeriana, Bogotá, Médico internista neumólogo, Hospital Universitario San Ignacio, Bogotá, Colombia Patricia Hidalgo-Martínez Departamento de Medicina Interna, Facultad de Medicina, Pontificia Universidad Javeriana, Bogotá, Médica internista neumóloga, Hospital Universitario San Ignacio, Bogotá, Colombia Mary Bermúdez Gómez

Departamento de Medicina Interna, Facultad de Medicina, Pontificia Universidad Javeriana, Bogotá, Médica internista neumóloga, Hospital Universitario San Ignacio, Bogotá, Colombia

a Autora de correspondencia: mbermude@javeriana.edu.co

Cómo citar: Torres-González JV, Botero JD, CelisPreciado CA, Fernández MJ, Villaquirán C, García OM, Solarte I, Hidalgo-Martínez P, Bermúdez Gómez M. Fibrosis pulmonar en infección por SARS-CoV-2: ¿qué sabemos hasta hora? ¿Qué podemos esperar? Univ. Med. 2020;61(4). https://doi.org/10.11144/Jave riana.umed61-4.fibr 


\section{RESUMEN}

La infección por SARS-CoV-2 le ha traído grandes retos al personal de salud durante 2020. Aprender sobre su comportamiento, fisiopatología, afectación pulmonar y su progresión a síndrome de dificultad respiratoria aguda (SDRA), además de las intervenciones farmacológicas y la mortalidad, han representado un desafío científico en los centros de atención especializada. No obstante, y a pesar de los avances sobre la infección aguda, poco se conoce respecto a las potenciales secuelas posteriores a la infección leve, moderada o severa por SARS-CoV-2, entre ellas la posible afectación fibrosante del parénquima pulmonar, que de acuerdo con la experiencia sobre infecciones por otros coronavirus (síndrome respiratorio agudo severo y síndrome respiratorio de Oriente Medio) y otras causas de SDRA, puede ser una de las complicaciones más severas y con mayor impacto en la calidad de vida y en la funcionalidad de las personas, una vez superada la infección. En este artículo se revisa la evidencia disponible sobre el desarrollo de la enfermedad pulmonar intersticial de tipo fibrosante asociada con la infección por SARS-CoV-2, los posibles mecanismos fisiopatológicos, los hallazgos imagenológicos y el plan de manejo propuesto hasta el momento, especialmente en términos de rehabilitación integral de estos pacientes.

Palabras clave

fibrosis pulmonar; SARS-CoV-2; COVID-19; rehabilitación pulmonar.

\begin{abstract}
SARS-CoV-2 infection has brought great challenges for health personnel during 2020. Learn about the behavior of the virus, the pathophysiology of the disease, lung involvement and its progression to respiratory distress syndrome (ARDS), in addition to pharmacological interventions and mortality have represented a scientific challenge in specialized care centers. However, despite the progress that has been made in acute infection, little is known about the potential sequelae after mild, moderate, or severe SARS-CoV-2 infection, including the possible fibrosing involvement of the lung parenchyma, which according to the previous experience on infections by other coronaviruses (SARS and MERS) and other causes of ARDS, it can be one of the most severe complications and with the greatest impact on the quality of life and functionality of people, once the infection is over. In this document, we present a review of the available evidence on the development of fibrosing-type interstitial lung disease associated with SARS-CoV-2 infection, possible pathophysiological mechanisms, imaging findings, and the management plan proposed so far, especially in terms of comprehensive rehabilitation of these patients.
\end{abstract}

Keywords

pulmonary fibrosis; SARS-CoV-2; COVID 19; pulmonary rehabilitation.

\section{Introducción}

La neumonía por SARS-CoV-2 supone un reto para el sistema inmune y, específicamente, para el pulmón. En el curso natural de la enfermedad se han identificado tres estadios: una primera fase, infecciosa, en la que la mayoría de los pacientes son asintomáticos; una fase intermedia, en la que se presenta insuficiencia pulmonar, dada por infiltrados en vidrio esmerilado e hipoxemia, y una tercera fase, hiperinflamatoria, en la que podemos ubicar a los pacientes más graves, con disfunción multiorgánica, trastornos de la coagulación y síndrome de dificultad respiratoria aguda (SDRA) (1). Anteriores coronavirus han sido relacionados con el desarrollo de fibrosis pulmonar, como en el caso del síndrome respiratorio de Oriente Medio (MERS) y el síndrome respiratorio agudo severo (SARS), con una mortalidad aproximada del 9,2\% (2).

Hasta la fecha (9 de septiembre de 2020), hemos tenido 680.000 casos confirmados en Colombia, con 541.000 personas recuperadas de COVID-19; pero todavía es incierta la magnitud de la afectación a largo plazo luego de superar la infección, específicamente para quienes han tenido neumonía grave y SDRA.

En el presente artículo revisamos la evidencia disponible con relación a la ocurrencia de fibrosis pulmonar posterior a la infección por SARS. CoV-2, ante la plausibilidad biológica de secuelas persistentes, que influyan en la función pulmonar y en la calidad de vida. La incertidumbre respecto al comportamiento del SARS-CoV-2 condiciona la necesidad de hacerles un seguimiento a estos pacientes y planear un programa de rehabilitación integral para asegurar la máxima recuperación en cada caso.

\section{¿Cuáles fueron las secuelas pulmonares del SARS?}

El coronavirus responsable del SARS en 8096 personas de 32 países durante la pandemia de 2002 tiene datos limitados para secuelas pulmonares, dado que no existen series a largo plazo con un volumen importante de 
pacientes, aun cuando se documentó que algunos se recuperaron de anormalidades funcionales pulmonares y radiológicas de diversos grados de severidad.

Estudios con pruebas de función pulmonar documentaron patrones con alteración restrictiva en la espirometría con capacidad vital forzada menores al $80 \%$ del predicho y disminución en las presiones inspiratoria máxima y espiratoria máxima, que se mantenían hasta 6 semanas posteriores al alta, con recuperación completa en el $80 \%$ de los casos al año de seguimiento. Estos hallazgos han sugerido la presencia de debilidad muscular como el factor principal para la alteración de la función pulmonar en estos pacientes, la cual pudo deberse a varios factores, entre los que se destacan uso de dosis altas de esteroides, reposo prolongado, desacondicionamiento físico o efecto sistémico residual de la enfermedad aguda (3).

Además, se identificó que en hasta el $30 \%$ de los pacientes había alteraciones en la capacidad de difusión del monóxido de carbono menores al $80 \%$ predicho que se recuperó aproximadamente luego de un año de seguimiento, en un $80 \%$ de los casos. Estos hallazgos sugieren que, con el tiempo, la afectación de la función pulmonar causada por el SARS podría mejorar de manera espontánea (3).

Por otro lado, estudios con tomografía de tórax axial computada de alta resolución (TACAR) mostraron que en el $62 \%$ de los pacientes hubo cambios consistentes con fibrosis pulmonar a las 5 semanas posteriores al alta. En estos, el tipo de fibrosis fue irregular, no extensa y sin impacto significativo sobre la función pulmonar (4). En otras cohortes se observó fibrosis pulmonar persistente: en una de ellas estuvo en el $21 \%$ de los pacientes a los 9 meses (5), y en otra, en el $28 \%$ a los 12 meses (6). La anormalidad radiográfica más frecuente en los supervivientes que cursaron con SDRA durante el primer mes de recuperación fue la existencia de opacidades en vidrio esmerilado, y en el control a los 3 y 6 meses se identificaron signos de fibrosis, entre estos infiltrados reticulares, en el $36 \%$ y el $30 \%$ de los pacientes, respectivamente (6).
En un estudio de seguimiento temprano de pacientes con SARS, 15 de 24 pacientes evidenciaron fibrosis pulmonar, con una duración media de seguimiento de 37 días después del alta hospitalaria (4). Los pacientes con mayor riesgo de desarrollar la fibrosis fueron mayores en edad y tuvieron más probabilidades de necesitar atención en la unidad de cuidado intensivo (UCI) que los pacientes sin fibrosis. En un estudio de seguimiento de 36 pacientes que sobrevivieron a la infección por el coronavirus del MERS, 12 tenían evidencia radiográfica de fibrosis pulmonar. Estos pacientes eran mayores y tenían ingresos más largos en las UCI.

Las anomalías predominantes en la tomografía axial computada en pacientes con SARS incluyeron opacidades de vidrio esmerilado, rápidamente progresivas, a veces con consolidación. Los cambios reticulares se notaron aproximadamente 2 semanas después del inicio de los síntomas y persistieron en la mitad de los pacientes después de las 4 semanas. Sin embargo, un estudio de seguimiento de 15 años a 71 pacientes con SARS mostró que las anormalidades intersticiales y el deterioro funcional se recuperaron durante los primeros 2 años después de la infección y luego se mantuvieron estables. A los 15 años, el 4,6\% de los pulmones mostraron anormalidad intersticial (3).

En pacientes con MERS, las anormalidades típicas de la tomografía axial computada incluyeron opacidades bilaterales de vidrio esmerilado, sobre todo en las zonas pulmonares basales y periféricas. Los resultados del seguimiento están menos descritos en pacientes con MERS. En un estudio de 36 pacientes que se habían recuperado de MERS, las radiografías de tórax tomadas con una mediana de 43 (rango 32-320) días después del alta hospitalaria mostraron anormalidades descritas como fibrosis pulmonar en aproximadamente un tercio de los pacientes. No se ha encontrado un seguimiento a largo plazo de pacientes que se recuperaron de MERS. 
July Vianneth Torres-González, Juan David Botero, Carlos Andrés Celis-Preciado, et al.

\section{Posibles mecanismos fisiopatológicos de fibrosis pulmonar en SARS}

La lesión epitelial alveolar y la formación de focos de miofibroblastos activos son el origen de la mayoría de los procesos fibróticos pulmonares $(7,8)$. Una vez que se produce daño en el tejido pulmonar, por cualquier agente nocivo, un conjunto de factores de crecimiento y citocinas es sobreexpresado y liberado por los neumocitos tipo II, que desencadenan la hiperproliferación de estas células, el reclutamiento de los fibroblastos a los núcleos fibróticos y su diferenciación en miofibroblastos. Son estos últimos los responsables de la acumulación de matriz extracelular en las membranas basales y tejidos intersticiales, lo que finalmente lleva a la pérdida de la función alveolar $(9,10)$.

Una de las moléculas más relevantes en el desarrollo de fibrosis es el factor de crecimiento transformante $B$ (TGF- $\beta$ ). Se trata de una citocina que actúa como regulador del crecimiento, diferenciación y muerte celular, así como antinflamatorio y estimulador de la migración y remodelación de la matriz extracelular. El TGF- $\beta$ incrementa la secreción de inhibidores de proteasa (PAI-1 y TIMP) y reduce la secreción de proteasa. Adicionalmente, induce la transición de las células epiteliales para formar fibroblastos y, por lo tanto, miofibroblastos a través de un proceso denominado transición epitelio-mesénquima (11-13).

En el caso de la infección por SARS-CoV-2 se documentaron concentraciones séricas elevadas de TGF- $B$ en las células epiteliales alveolares, las epiteliales bronquiales, los monocitos y los macrófagos. El mecanismo preciso es desconocido; sin embargo, se postula un mecanismo de regulación vinculado con los niveles del receptor 2 de la enzima convertidora de angiotensina (ECA2). El TGF- $\beta$ sérico, que indujo la proliferación de fibroblastos, se sobreexpresó en la etapa tardía de la infección por coronavirus $(14,15)$.

El receptor ECA2 es un componente del sistema renina-angiotensina, esencial para la infección por el SARS-CoV-2, dado que la proteína $S$ del virus se une a él en la superficie celular, lo que permite la entrada viral en la célula. Esto hiperactiva el sistema reninaangiotensina con regulación negativa de ECA2 y disminución de las cantidades circulantes de angiotensina II, por un aumento de su acción patogénica al actuar sobre los receptores AT1, que induce el TFG- $\beta$ implicado en la inflamación pulmonar, cardiaca y renal $(16,17)$.

El aumento en las cantidades de TFG- $\beta$ se considera el principal mecanismo responsable del desarrollo de fibrosis pulmonar y está implicado en otras enfermedades pulmonares de larga duración, como hipertensión arterial pulmonar. Así mismo, el descenso en la ECA2 lleva a un incremento profibrótico, proliferativo y vasoactivo en los casos más severos. Así mismo, se ha sugerido que la fibrosis pulmonar en el SARS-CoV-2 la causa una respuesta hiperactiva del hospedero a la lesión pulmonar mediada por la señalización del receptor del factor de crecimiento epidérmico.

Ding et al. (18) analizaron de forma retrospectiva a 32 pacientes con diagnóstico de neumonía por SARS-CoV-2 leve (11 pacientes), severa (10 pacientes) y crítica (11 pacientes). Las concentraciones séricas de ácido hialurónico y procolágeno tipo III detectadas al ingreso distinguieron claramente a los pacientes críticos, y las de laminina discriminaron el grupo crítico y del grupo leve. Estos marcadores se han relacionado con reparación tisular y fibrosis.

Se ha argumentado que el proceso inflamatorio disregulado durante el SDRA puede fomentar la proliferación fibroblástica y un posterior desarrollo de fibrosis pulmonar. No obstante, en la mayoría de los estudios de seguimiento que han incluido medidas fisiológicas y escanografía de tórax, las anomalías radiográficas persistentes después del SDRA tienen poca relevancia clínica y se han vuelto menos comunes en la era de la ventilación pulmonar protectora $(19,20)$.

Por otra parte, un metanálisis llevado a cabo por Gaohong et al. (citado en 21) incluyó 20 estudios de casos y controles con 1287 participantes. Señaló que las infecciones virales aumentan casi cuatro veces el riesgo 
de desarrollar fibrosis pulmonar idiopática (OR: 3,48; IC95 \%: 1,61-7,52; $\mathrm{p}=0,001)$.

\section{Función pulmonar tras neumonía por SARS-COV-2}

Mo et al. (22), teniendo en cuenta publicaciones acerca de la alteración persistente de la función pulmonar y la capacidad de ejercicio posterior a la infección por otros coronavirus (MERS y SARS), evaluaron la función pulmonar posterior al egreso en sujetos con diagnóstico confirmado de infección por SARS-CoV-2 no crítica (definida como requerimiento de ventilación mecánica, choque séptico o falla de otro órgano). Se incluyeron 24 casos leves, 67 casos con neumonía y 19 con neumonía severa. El promedio de edad fue de 49 años y el $40 \%$ tenía, al menos, una comorbilidad. Se encontraron anormalidades en la capacidad de difusión de monóxido de carbono en 51 sujetos (47,2\%); en la capacidad pulmonar total evaluada por volúmenes pulmonares en un $25 \%$, compatible con un trastorno restrictivo; en el volumen espiratorio forzado en un segundo, evaluado por espirometría en el 13,6\%, y en la capacidad vital forzada, en un 9,1\%. Dichas anormalidades estuvieron presentes en un 30,4 $\%$ en sujetos con enfermedad leve, en un $42,4 \%$ en aquellos que cursaron con neumonía y en un $84,2 \%$ de los que tuvieron neumonía severa ( $\mathrm{p}$ $<0,05)$.

Es importante resaltar que el estudio tiene limitaciones, como la falta de una evaluación basal de la función pulmonar y la falta de correlación entre el compromiso imagenológico y la función pulmonar, y al tratarse de un análisis de corte transversal, no se evaluaron las dinámicas de la variación a largo plazo (22).

\section{Evidencia de la presencia de fibrosis pulmonar tras COVID-19 en TACAR}

Para el 1. de junio de 2020 se habían publicado cinco estudios que evaluaban la presencia de fibrosis pulmonar en la TACAR de tórax de pacientes con neumonía por SARS-CoV-2 (tabla 1).
Tabla 1.

Estudios que han evaluado la presencia de fibrosis pulmonar en TACAR de tórax de seguimiento en pacientes con neumonía por SARS-CoV-2

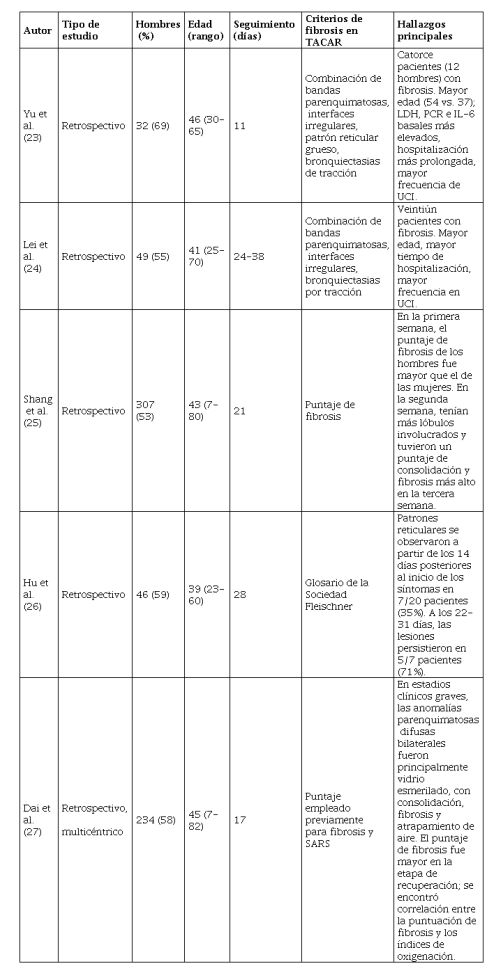

Dichos trabajos se caracterizan por tener un diseño retrospectivo, incluir un número variado de pacientes (desde 32 a 234), en su mayoría hombres (53-69\%) y con edad promedio de 43 años (rango 7 a 82). El seguimiento de los participantes ha sido corto (11-38 días) y se han utilizado diversos criterios para la identificación de fibrosis pulmonar, desde hallazgos aislados o combinados como reticulaciones gruesas y bronquiectasias por tracción hasta puntajes de fibrosis utilizados en la literatura.

Los hallazgos, que deben ser considerados preliminares teniendo en cuenta lo ya mencionado, señalan que la presencia de fibrosis pulmonar después de la tercera semana de seguimiento se observó con mayor frecuencia en hombres, mayores de 50 años, con antecedente de ingreso a cuidado intensivo y hospitalización prolongada y con biomarcadores basales de inflamación elevados (28). 
La frecuencia de fibrosis pulmonar tras neumonía por SARS-COV-2 aún no puede determinarse con claridad; pero parecería ser más frecuente en los pacientes que han cursado con neumonía grave o SDRA.

\section{Tratamiento para fibrosis pulmonar en SARS-CoV-2}

Hasta no tener claro cuál es el panorama de desarrollo de fibrosis pulmonar en pacientes con neumonía por SARS-COV-2, es necesario ser cautos frente a la postulación de intervenciones farmacológicas.

Se describe que, en algunos casos, el uso de antifibróticos (inhibidores de tirosina cinasa como nintedanib), inmunomoduladores, antivirales inmunoupresores y terapia biológica usados en otras patologías fibrosantes del pulmón también podrían ser útiles para SARS-CoV-2 durante el SDRA o posteriormente (cuando se desarrolle la fibrosis pulmonar), por el hecho de haberse demostrado beneficio en otros escenarios (29), aunque hay reportes de efecto-beneficio con el uso de algunos de estos en los cambios fibróticos posteriores (30). Sin embargo, para esto se debe establecer la evaluación de dichos medicamentos en estudios clínicos, y más si se considera que no todos los episodios de inflamación y fibrosis van a terminar con fibrosis irreversible $(31,32)$.

Por su parte, Song y Shi (33) sugieren que la neumonía por SARS-CoV-2 puede tener una fisiopatología similar a la de la neumonitis de hipersensibilidad y, por esta razón, postulan que el uso de esteroides en etapas tempranas de la infección, en dosis altas de acuerdo con la severidad, podrían tener un rol importante en la disminución de la mortalidad y de la fibrosis pulmonar.

\section{Trasplante para fibrosis pulmonar}

En pacientes con SARS-COV-2 crítico, quienes cursan con SDRA, se ha descrito una mayor afectación estructural y funcional pulmonar; de ahí que el trasplante pulmonar se convierta en una opción terapéutica. En la literatura sobre el tema se ha descrito el trasplante de dos pacientes por fibrosis pulmonar relacionada con SARS$\mathrm{CoV}-2$, aunque es necesario aclarar que dicha fibrosis fue en el contexto de SDRA severo con persistencia de falla multiorgánica por alrededor de un mes. Tres pacientes se sometieron a este procedimiento y sobrevivieron dos, por lo que podría considerarse una alternativa para aquellas personas en estadios terminales de la falla respiratoria por SARS-CoV-2 (34).

\section{Rehabilitación pulmonar}

La rehabilitación pulmonar ha mostrado ser una intervención no farmacológica muy efectiva en el manejo integral de los pacientes con enfermedades respiratorias crónicas en estado estable y agudizado, pues mejora los síntomas y la calidad de vida y reduce el riesgo de exacerbaciones (35). Esta es una intervención individualizada que utiliza el ejercicio aeróbico y de fuerza, junto con la educación, el soporte nutricional y el psicológico. Esta opción terapéutica se convierte en una alternativa en aquellos sujetos con enfermedad grave con insuficiencia pulmonar irreversible.

A partir de la evidencia de la infección por SARS, se conoce que pacientes con infecciones graves, tiempos prolongados en cama, cambios patológicos residuales como atelectasias, fibrosis y grados variables de debilidad o disfunción muscular (36), la rehabilitación pulmonar fue de gran ayuda.

Lau et al. (37) condujeron un programa de rehabilitación pulmonar durante 6 semanas para 133 pacientes. Incluyó sesiones de 30 minutos de ejercicio aeróbico, seguido de sesiones de resistencia en miembros inferiores y educación. Comparado con el grupo control, se encontró una mejoría estadísticamente significativa en la caminata de 6 minutos, la cual evalúa la capacidad de ejercicio y el consumo de oxígeno en la prueba de resistencia.

En la infección por SARS-CoV-2 se ha considerado que el objetivo a corto plazo de la rehabilitación pulmonar debe ser aliviar la disnea, 
la ansiedad y la depresión; entre tanto, a largo plazo, los objetivos son mejorar la calidad de vida, facilitar el retorno a la vida en sociedad y preservar la función pulmonar (38). Será necesaria una evaluación individualizada previa al inicio del programa de rehabilitación, al igual que un análisis de los riesgos clínicos y los derivados del ejercicio. Y como resultado de esa evaluación se deberá combinar el entrenamiento con ejercicio de resistencia, fuerza muscular, balance y flexibilidad.

La guía de la British Thoracic Society, publicada en 2020, recomienda como parte del manejo posterior a la neumonía por SARSCoV-2 referir a programas de rehabilitación pulmonar (39). Por otra parte, en el contexto de la actual pandemia, la Asociación Americana del Tórax sugiere ofrecer programas de rehabilitación domiciliaria con supervisión en tiempo real (teleorientación), intervención que en otros escenarios ha mostrado efectividad y seguridad (35).

\section{Conclusiones}

La fibrosis pulmonar puede desarrollarse después de una inflamación crónica o como un proceso fibroproliferativo primario, genéticamente influenciado y relacionado con la edad y es una secuela reconocida tras un SDRA.

Sin embargo, la mayoría de los estudios de seguimiento, que han incluido medidas fisiológicas y radiológicas, han demostrado que las anomalías radiográficas persistentes después del SDRA tienen poca relevancia clínica y se han vuelto menos comunes en la era de la ventilación pulmonar protectora. Igualmente, con el tiempo, algunos pacientes recuperan su función pulmonar.

Teniendo en cuenta las experiencias previas con el SARS y lo observado hasta ahora con el SARS-CoV-2, se estima que alrededor del $40 \%$ de los pacientes con infección por SARS-CoV-2 desarrollan SDRA y que el $20 \%$ de los casos de SDRA son graves (40). Cabe destacar que la edad promedio de los pacientes hospitalizados con infección por SARS-CoV-2 grave parece ser mayor que la observada con MERS o SARS y que la edad avanzada es un factor de riesgo para el desarrollo de fibrosis pulmonar. Dadas estas observaciones, podría ser sustancial la carga de fibrosis pulmonar después de la recuperación de SARS-CoV-2.

En la actualidad, las consecuencias pulmonares a largo plazo de la neumonía por SARS-CoV-2 siguen siendo especulativas y no deben asumirse sin un estudio prospectivo adecuado. No obstante, dada la gran cantidad de individuos afectados por SARS-CoV-2, incluso las complicaciones raras tendrán efectos importantes en la salud de la población. Es importante identificar rápidamente si el desarrollo de fibrosis pulmonar ocurre en la población sobreviviente. Con esto, podemos esperar brindar la atención clínica adecuada y diseñar programas de seguimiento cercano de la función pulmonar, los síntomas, la funcionalidad y calidad de vida, que permitan a su vez trabajar en ensayos de intervención para prevenir una segunda ola de morbilidad y mortalidad tardía asociada con esta devastadora pandemia.

\section{Referencias}

1. Mason RJ. Pathogenesis of COVID-19 from a cell biology perspective. Eur Respir J. 2020;55(4):2000607. https://doi.org/1 0.1183/13993003.00607-2020

2. Tse GM, To KF, Chan PK, Lo AW, Ng KC, Wu A, et al. Pulmonary pathological features in coronavirus associated severe acute respiratory syndrome (SARS). J Clin Pathol. 2004;57 (3):260-5. https://doi.org/10.1 136/jcp.2003.013276

3. Xie LX, Liu YN, Fan BX, Xiao YY, Tian Q, Chen LG, et al. Dynamic changes of serum SARSCoronavirus IgG, pulmonary function and radiography in patients recovering from SARS after hospital discharge. Respir Res. 2005;6:5. https://doi.org/10 $.1186 / 1465-9921-6-5$ 
4. Antonio GE, Wong KT, Hui DS, Wu A, Lee N, Yuen EH, et al. Thin-section CT in patients with severe acute respiratory syndrome following hospital discharge: preliminary experience. Radiology. 2003;228:810-5. https://doi. org/10.1148/radiol.2283030726

5. Hwang DM, Chamberlain DW, Poutanen SM, Low DE, Asa SL, Butany J. Pulmonary pathology of severe acute respiratory syndrome in Toronto. Mod Pathol. 2005;18:1-10.

6. Hui DS, Wong KT, Ko FW, Tam LS, Chan DP, Woo J, Sung JJY. The 1-year impact of severe acute respiratory syndrome on pulmonary function, exercise capacity, and quality of life in a cohort of survivors. Chest. 2005;128:2247-2261.

7. Chapman HA. Disorders of lung matrix remodeling. J Clin Invest. 2004;113:148-57.

8. Gharaee-Kermani M, Gyetko MR, $\mathrm{Hu}$ B, Phan SH. New insights into the pathogenesis and treatment of idiopathic pulmonary fibrosis: a potential role for stem cells in the lung parenchyma and implications for therapy. Pharm Res. 2007;24:819-41.

9. Zuo W, Zhao X, Chen Y. SARS coronavirus and lung fibrosis. Molecular biology of the SARS coronavirus. 2009:247-58. https://doi.o rg/10.1007/978-3-642-03683-5_15

10. Chapter 15: SARS Coronavirus and Lung Fibrosis. En: Molecular biology by the SARS-Coronavirus. Heidelberg: Springer; 2010. p. 247-58. https://doi.org/10.1007/978-3642-03683-5.

11. Derynck R, Miyazono K. The TGF-beta family. Cold Spring Harbor Laboratory; 2008.

12. Rube CE, Uthe D, Schmid KW, Richter KD, Wessel J, Schuck A, Willich N, Rube C. Dosedependent induction of transforming growth factor beta (TGF-beta) in the lung tissue of fibrosis-prone mice after thoracic irradiation. Int J Radiat Oncol Biol Phys. 2000;47:1033-42.

13. Derynck R, Akhurst RJ. Differentiation plasticity regulated by TGF-beta family proteins in development and disease. Nat Cell Biol. 2007;9:1000-4.

14. Pang BS, Wang Z, Zhang LM, Tong ZH, Xu LL, Huang XX, et al. Dynamic changes in blood cytokine levels as clinical indicators in severe acute respiratory syndrome. Chin Med J. 2003;116:1283-7.

15. Baas $\mathrm{T}$, Taubenberger JK, Chong PY, Chui P, Katze MG. SARS-CoV virus-host interactions and comparative etiologies of acute respiratory distress syndrome as determined by transcriptional and cytokine profiling of formalin-fixed paraffin-embedded tissues. J Interferon Cytokine Res. 2006;26:309-17.

16. Kuba K, Imai Y, Penninger JM. Angiotensin-converting enzyme 2 in lung diseases. Curr Opin Pharmacol. 2006;6:271-6.

17. González-Jaramillo N, Low N, Franco $\mathrm{OH}$. The double burden of disease of COVID-19 in cardiovascular patients: overlapping conditions could lead to overlapping treatments. Eur J Epidemiol. 2020;35:335-7.

18. Ding $M$, Zhang Q, Li Q, Wu T, Huang Y-Z. Correlation analysis of the severity and clinical prognosis of 32 cases of patients with COVID-19. Respirat Med. 2020;167. https://doi.org/10.1016/j.rmed.2020. 105981

19. Marshall R, Bellingan G, Laurent G. The acute respiratory distress syndrome: fibrosis in the fast lane. Thorax. 1998;53(10):815-7. 
20. Burnham EL, Janssen WJ, Riches DWH, Moss M, Downey GP. The fibroproliferative response in acute respiratory distress syndrome: mechanisms and clinical significance. Eur Respir J. 2014;43(1):276-85.

21. Sheng G, Chen P, Wei Y, Yue H, Chu J, Zhao J, et al. Viral infection increases the risk of idiopathic pulmonary fibrosis: a meta-analysis. Chest. 2020;157(5):1175-87. https://d oi.org/10.1016/j.chest.2019.10.032

22. Mo X, Jian W, Su Z, Chen M, Peng $\mathrm{H}$, Peng $\mathrm{P}$, et al. Abnormal pulmonary function in COVID-19 patients at time of hospital discharge. Eur Respir J. 2020;55(6):2001217. https://doi.org/1 0.1183/13993003.01217-2020

23. Yu M, Liu Y, Xu D, Zhang R, Lan $\mathrm{L}, \mathrm{Xu}$ H. Prediction of the development of pulmonary fibrosis using serial thinsection CT and clinical features in patients discharged after treatment for COVID-19 pneumonia. Korean J Radiol. 2020 Jun;21(6):746-55. https:/ /doi.org/10.3348/kjr.2020.0215

24. Lei P, Fan B, Mao J, Wei J, Wang P. The progression of computed tomographic (CT) images in patients with coronavirus disease (COVID-19) pneumonia. J Infect. 2020 Jun;80 (6):e30-1. https://doi.org/1 0.1016/j.jinf.2020.03.020

25. Shang $\mathrm{Y}, \mathrm{Xu} \mathrm{C}$, Jiang F, Huang R, Li Y, Zhou Y, Xu F, Dai H. Clinical characteristics and changes of chest CT features in 307 patients with common COVID-19 pneumonia infected SARS-CoV-2: A multicenter study in Jiangsu, China. Int J Infect Dis. 2020 May 8;96:157-62. https://doi.org/ 10.1016/j.ijid.2020.05.006

26. Hu Q, Guan H, Sun Z, Huang L, Chen C, Ai T, Pan Y, Xia L. Early CT features and temporal lung changes in COVID-19 pneumonia in Wuhan, China. Eur J Radiol. 2020
Apr 19;128:109017. https://doi.org/10. 1016/j.ejrad.2020.109017

27. Dai H, Zhang X, Xia J, Zhang T, Shang Y, Huang R, Liu R, Wang D, Li M, Wu J, Xu Q, Li Y. High-resolution Chest CT features and clinical characteristics of patients infected with COVID-19 in Jiangsu, China. Int J Infect Dis. 2020 Apr 6;95:106-11.

28. Hosseiny M, Kooraki S, Gholamrezanezhad A, Reddy S, Myers L. Radiology perspective of coronavirus disease. 2019 (COVID-19): lessons from severe acute respiratory syndrome and middle east respiratory syndrome. Am J Roentgenol. 2020;214(5):1078-82. https://doi.org/1 0.2214/AJR.20.22969

29. George PM, Wells AU, Jenkins RG. Pulmonary fibrosis and COVID-19: the potential role for antifibrotic therapy. Lancet Respir Med. Mayo de. 2020;S2213260020302253.

30. Saha A, Vaidya PJ, Chavhan VB, Achlerkar A, Leuppi JD, Chhajed PN. Combined pirfenidone, azithromycin and prednisolone in post-H1N1 ARDS pulmonary fibrosis. Sarcoidosis Vasc Diffuse Lung Dis. 2018;35:85-90.

31. Chang C-H, Juan Y-H, Hu HC, Kao K-C, Lee C-S. Reversal of lung fibrosis: an unexpected finding in survivor of acute respiratory distress syndrome. QJM Int J Med. 1 de enero de. 2018;111(1):47-8. https://doi.org/1 0.1093/qjmed/hcx190

32. Rockey DC, Bell PD, Hill JA. Fibrosis: a common pathway to organ injury and failure. $\mathrm{N}$ Engl $\mathrm{J}$ Med. 2015;372(12):1138-49.

33. Song YG, Shin HS. COVID-19: a clinical syndrome manifesting as hypersensitivity pneumonitis. Infect Chemother. 2020 Mar;52(1):110-2. ht tps://doi.org/10.3947/ic.2020.52.1.110 
34. Chen J-Y, Qiao K, Liu F, Wu $\mathrm{B}$, $\mathrm{Xu} \mathrm{X}$, Jiao G-Q, et al. Lung transplantation as therapeutic option in acute respiratory distress syndrome for COVID-19-related pulmonary fibrosis: Chin Med J (Engl). 2020;1.

35. Spruit MA, Singh SJ, Garvey C, ZuWallack R, Nici L, Rochester C, et al. An official American Thoracic Society/European Respiratory Society statement: key concepts and advances in pulmonary rehabilitation. Am J Respir Crit Care Med. 2013;188(8):e13-64.

36. Herridge MS, Cheung AM, Tansey CM, Matte-Martyn A, Diaz-Granados $\mathrm{N}, \mathrm{Al}$-Saidi F, et al. One-year outcomes in survivors of the acute respiratory distress syndrome. N Engl J Med. 2003;348(8):683-93. https://doi.org/10 $.1056 /$ NEJMoa022450

37. Lau HM, Ng GY, Jones AY, Lee EW, Siu EH, Hui DS. A randomised controlled trial of the effectiveness of an exercise training program in patients recovering from severe acute respiratory syndrome. Aust J Physiother. 2005;51(4):213-9. https://doi.org/10.1 016/s0004-9514(05)70002-7

38. Yang L-L, Yang $T$. Pulmonary rehabilitation for patients with coronavirus disease 2019 (COVID-19). Chronic Dis Transl Med. 2020;6(2):79-86. https://doi.org/10.10 16/j.cdtm.2020.05.002

39. British Thoracic Society. British Thoracic Society guidance on respiratory follow up of patients with a clinico-radiological diagnosis of COVID-19 pneumonia. 2020 may 11.

40. Spagnolo P, Balestro E, Aliberti $S$, Cocconcelli E, Biondini D, Casa GD, et al. Pulmonary fibrosis secondary to COVID-19: a call to arms? Lancet Respir Med.
2020;8(8):750-2. https://doi.org/10.10

$16 / S 2213-2600(20) 30222-8$ 